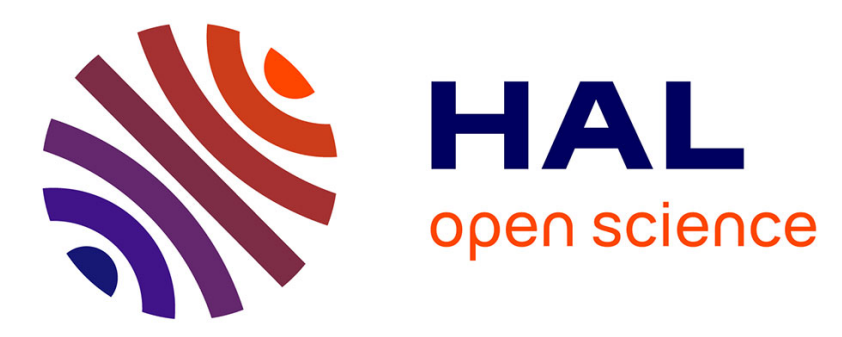

\title{
Influence of the chemical modeling on the quenching limits of gaseous detonation waves confined by an inert layer
}

Said Taileb, J. Melguizo-Gavilanes, Ashwin Chinnayya

\section{- To cite this version:}

Said Taileb, J. Melguizo-Gavilanes, Ashwin Chinnayya. Influence of the chemical modeling on the quenching limits of gaseous detonation waves confined by an inert layer. Combustion and Flame, 2020, 218, pp.247 - 259. 10.1016/j.combustflame.2020.04.018 . hal-02989902

\section{HAL Id: hal-02989902 \\ https://hal.science/hal-02989902}

Submitted on 5 Nov 2020

HAL is a multi-disciplinary open access archive for the deposit and dissemination of scientific research documents, whether they are published or not. The documents may come from teaching and research institutions in France or abroad, or from public or private research centers.
L'archive ouverte pluridisciplinaire HAL, est destinée au dépôt et à la diffusion de documents scientifiques de niveau recherche, publiés ou non, émanant des établissements d'enseignement et de recherche français ou étrangers, des laboratoires publics ou privés. 


\title{
Influence of the chemical modeling on the quenching limits of gaseous detonation waves confined by an inert layer
}

\author{
S. Taileb*, J. Melguizo-Gavilanes, A. Chinnayya \\ Institut Pprime, UPR 3346 CNRS, ISAE-ENSMA, Université de Poitiers, 86961 \\ Futuroscope-Chasseneuil, France
}

\begin{abstract}
The effect of chemistry modeling on the flow structure and quenching limits of detonations propagating into reactive layers bounded by an inert gas is investigated numerically. Three different kinetic schemes of increasing complexity are used to model a stoichiometric $\mathrm{H}_{2}-\mathrm{O}_{2}$ mixture: single-step, three-step chainbranching and detailed chemistry. Results show that while the macroscopic characteristics of this type of detonations e.g. velocities, cell-size irregularity and leading shock dynamics, are similar among the models tested, their instantaneous structures are significantly different before and upon interaction with the inert layer when compared using a fixed height. When compared at their respective critical heights, $h_{\text {crit }}$, i.e. the reactive layer height at which successful detonation propagation is no longer possible, similarities in their structures become apparent. The numerically predicted critical heights increase as $h_{\text {crit, Detailed }} \ll h_{\text {crit, 3-Step }}<$ $h_{\text {crit, } 1 \text {-Step }}$. Notably, $h_{\text {crit, Detailed }}$ was found to be in agreement with experimentally reported values. The physical mechanisms present in detailed chemistry and ne-
\end{abstract}

\footnotetext{
*Corresponding author: said.taileb@ensma.fr
} 
glected in simplified kinetics, anticipated to be responsible for the discrepancies obtained, are discussed in detail.

Keywords: Detonation dynamics, chemistry modeling, simplified kinetics, detailed chemistry, quenching limits, numerical simulation

\section{Introduction}

A detonation is a combustion front coupled with a strong leading shock wave that travels supersonically with respect to reactants at rest. Its propagation mechanism can be summarized as follows: (i) the shock wave compresses and heats up fresh reactants; (ii) due to the high temperature sensitivity of the chemistry this abrupt temperature increase results in very fast chemical reactions; and (iii) the volumetric expansion of the burnt gases across the reaction zone drives the leading shock wave forward. A strong coupling between the leading shock wave and the reaction zone is a key feature of self-sustained detonation waves [1]. The one-dimensional laminar structure of a detonation is then composed of an induction length, $l_{\text {ind }}$ (i.e. the distance between the leading shock and the start of heat release), and a reaction zone across which temperature increases and density decreases resulting in flow expansion until the flow velocity reaches the speed of sound in burnt products in a frame of reference attached to the shock, i.e. sonic point. The distance between the leading shock and the sonic plane is typically referred to as the hydrodynamic thickness, $h_{t}[2,3]$.

In reality, detonations exhibit an unsteady multidimensional cellular structure that departs significantly from its laminar description [4] drawn by the triple points formed by the interaction of the leading shock, and transverse waves traveling along the height of the wave. As a result, $l_{\text {ind }}$ and $h_{t}$ (characteristic length scales 
of detonations) become dynamic entities. Any disturbance coming towards the leading shock and reaction zone complex from regions downstream of the hydrodynamic thickness will not affect the dynamics of the detonation wave. However, it remains vulnerable to the influence of the boundaries confining the wave. The nature of these boundaries and their length scale ratios to the detonation cell size, $\lambda$, and/or $h_{t}$, result in a detonation velocity deficit, defined as a percentage of the ideal detonation propagation velocity (Chapman-Jouguet - CJ), $D / D_{\mathrm{CJ}}$, and eventual quenching. Various boundary conditions have been investigated in literature. They can be classified in two main categories: (i) smooth boundaries, such as constant cross section tubes [5], divergent channels [6] and yielding confinements [7]; and (ii) rough boundaries, such as tubes with obstacles [8] and porous walls [9]. The main failure mechanisms described are lateral expansion waves and attenuation of transverse waves that lead to the decoupling of the leading shock wave and the reaction zone. Experimental evidence shows that the quenching process differs depending on the mixture regularity. In [9] the critical dimension for detonation propagation in porous walls was determined to be $11 \lambda$ and $4 \lambda$, for mixtures exhibiting a regular and irregular cellular structures, respectively. This outcome was attributed to stronger transverse waves that are able to compensate for the losses, hence rendering the detonation more resilient to failure.

Another relevant boundary condition comes from the presence of compressible confinement $[10,11,12,13]$. In this configuration, the detonation front propagates in a layer of reactive mixture bounded at the top by inert gas. Lateral expansion of the products, streamline divergence, as well as triple point absorption/reflection at the interface lead to a very complex detonation front structure whose resulting curvature has been recently argued to be the main energy loss 
mechanism [14]. This canonical configuration also has implications for propulsion applications such as Rotating Detonation Engines, and safety. For the former, understanding non-idealities and predicting the detonation quenching limits is of prime importance for optimization and practical implementation of these devices. For the latter, understanding the mechanisms that lead to quenching and re-ignition is vital to mitigate the consequences of accidental leaks in industries such as nuclear, chemical processing plants, among others [15].

Classical experiments using the latter configuration were carried out using a rectangular cross-section composed of three rigid walls and a film separating reactive and inert mixtures $[16,17,18]$. Schlieren visualization revealed the main features of the wave complex which consist of a curved detonation front coupled to a transmitted oblique shock wave in the inert gas and a mixing layer separating shocked inert gas from hot detonation products. Special attention was given to the velocity deficit as a function of the height of the channel, the reactive layer height and the acoustic impedance ratio of inert to reactive gas. The authors found that the velocity deficit is proportional to the reactive layer height. Furthermore, the acoustic impedance was found to influence the structure of the propagating wave complex. As the confinement becomes lighter, i.e. lower acoustic impedance, the oblique shock in the inert gas detaches and moves ahead of the detonation. The critical height, $h_{\text {crit }}$, obtained for stoichiometric $\mathrm{H}_{2}-\mathrm{O}_{2}$ bounded by $\mathrm{N}_{2}$ was $3 \lambda$, and corresponded to a velocity deficit of $10 \%\left(D / D_{\mathrm{CJ}}=0.9\right)$ prior to quenching.

Murray and Lee [19] showed that transition from a rigid tube to a yielding confinement undergoes a first transient with partial quenching and re-ignition followed by steady propagation. The transition phase is characterized by generation of the ignition centers at the interface which sweep between the shock and the de- 
coupled reaction zone to re-establish the detonation wave. Quenching occurs if the detonation is not able to overcome the expansion losses coming from the presence of the interface. Experiments conducted by Rudy et al. [15, 20, 21] and Grune et al. [22] to determine the propagation limits of detonations in $\mathrm{H}_{2}-\mathrm{O}_{2}$ and $\mathrm{H}_{2}$-Air using the same experimental configuration as Dabora et al. [18] confirmed their findings, namely a $h_{\text {crit }} \sim 3 \lambda$ for stoichiometric $\mathrm{H}_{2}$-Air, and $2.4 \lambda \leq h_{\text {crit }} \leq 3.6 \lambda$ for $\mathrm{H}_{2}-\mathrm{O}_{2}$.

Reynaud et al. [10,11] and Mi et al. [13] carried out numerical simulations of detonation propagation into a reactive gas bounded by an inert gas for mixtures exhibiting regular and irregular cellular structures. Single-step chemistry with the same thermodynamic and kinetic parameters for the inert and reactive mixtures were used in both studies. The inert confinement influences the detonation propagation differently depending on the mixture regularity, and more importantly, $h_{\text {crit }}$, was found to be lower for regular than irregular mixtures, characterized by low and high activation energies, respectively. These results are in disagreement with the experimental observations in [9]; the discrepancy can be attributed to the use of inviscid models that fail to capture the mixing and burning of shocked unburnt mixture left behind the leading shock as argued in [23]. The presence of unburnt pockets is a characteristic feature of the structure of irregular detonations [24]. However, all the numerical work known to the authors and cited in [23] share an extra common assumption apart from an inviscid description of the flow: the chemistry is modeled assuming that the fuel is directly converted into products following a single irreversible reaction. When attempting to numerically predict quenching limits in real systems, in addition to the competition between cooling due to expansion and chemical heat release, the production/consumption 
of intermediates (active radicals) could also play an important role in extending or reducing the detonation propagation envelopes.

To gain some insight into the role of chemical modeling on the prediction of quenching limits, the present work uses three different models of increasing complexity: single-step, three-step chain-branching and detailed chemistry, to assess their effect on $h_{\text {crit }}$ for detonation propagation in a semi-confined environment. The detonation front dynamics and the two-dimensional structure of the front during steady propagation and quenching are examined.

\section{Computational methodology}

\subsection{Governing equations}

The flow is assumed to be inviscid and non-conducting hence described by the 2-D compressible Euler equations.

$$
\begin{aligned}
& \frac{\partial \rho}{\partial t}+\frac{\partial \rho u_{i}}{\partial x_{i}}=0 \\
& \frac{\partial \rho u_{i}}{\partial t}+\frac{\partial\left(\rho u_{i} u_{j}+p \delta_{i j}\right)}{\partial x_{i}}=0 \\
& \frac{\partial \rho e_{t}}{\partial t}+\frac{\partial\left(\rho u_{i}\left(e_{t}+p / \rho\right)\right)}{\partial x_{i}}=0 \\
& \frac{\partial \rho Y_{k}}{\partial t}+\frac{\partial \rho u_{i} Y_{k}}{\partial x_{i}}=\dot{\omega}_{k}, \quad k=1, \ldots, N
\end{aligned}
$$

where $\rho, u, p, e_{t}, Y_{k}, \dot{\omega}_{k}$ are the density, velocity, pressure, specific total energy, the mass fraction of species $k$ in a mixture composed of $N$ species, and the net production/consumption of species $k$, respectively. $\delta_{i j}$ is the Kronecker delta, and $x_{i}$ are the horizontal and vertical components of space with $i=1,2$, respectively. 
The total energy is expressed as

$$
e_{t}=e_{s}+\frac{1}{2} u_{i} u_{i}+q
$$

which includes the sensible internal energy, $e_{s}$, the kinetic energy, $\frac{1}{2} u_{i} u_{i}$, and the local heat release, $q$. The system of equations above is closed with an equation of state following the ideal gas law, $p=\rho \bar{R} T$, where $\bar{R}=R_{u} / W$ is the specific gas constant, $R_{u}$ the universal gas constant, $W$ the molecular weight of the mixture, and $T$ is the gas temperature. The expressions for $e_{s}$ and $q$ are dependent on the chemical modeling used.

\subsection{Chemistry modeling}

\subsubsection{Single-step kinetics}

The fuel, $\mathcal{F}$, is directly converted into products $\mathcal{P}$ following a single irreversible Arrhenius reaction:

$$
\mathcal{F} \stackrel{A_{s}}{\longrightarrow} \mathcal{P}
$$

The species conservation equation for the fuel, $Y_{\mathcal{F}}$, is:

$$
\frac{\partial \rho Y_{\mathcal{F}}}{\partial t}+\frac{\partial \rho u_{i} Y_{\mathcal{F}}}{\partial x_{i}}=\dot{\omega}_{\mathcal{F}}=-\rho Y_{\mathcal{F}} A_{s} \exp \left(-E_{a} / R_{u} T\right)
$$

$A_{s}$ and $E_{a}$ are the pre-exponential factor and the activation energy, respectively. The mixture is assumed to have a constant ratio of specific heats, $\gamma$, whose sensible internal energy and local heat release are given by $e_{s}=p /[(\gamma-1) \rho]$, and $q=$ $-\left(1-Y_{f}\right) Q ; Q$ is the energy content of the mixture.

\subsubsection{Three-step chain-branching kinetics}

In reality, chemical conversion of reactants into products does not take place in a single step, but through a sequence of intermediate stages which typically include chain-initiation, chain-branching and termination steps [25]. Therefore, we 
account for a few elementary reactions using a three-step chain-branching scheme originally proposed in [26]. To retain the essential dynamics of a real chainbranching reaction, the model consists, at first, of a thermally neutral initiation step with high activation energy in the Arrhenius term, which produces a small concentration of chain-carriers, $\mathcal{R}$, from the fuel, $\mathcal{F}$. Followed by a branchingstep, also thermally neutral and of Arrhenius type, with lower activation energy to allow for the concentration of chain-carriers to increase rapidly. Finally, an exothermic termination step in which chain-carriers are converted into products, $\mathcal{P}$, at a constant rate. Note that heat release could be associated with any/all steps, however here, heat release is only associated with the termination step. This assumption is a good approximation for $\mathrm{H}_{2}$ mixtures, whereas for some hydrocarbons, e.g. acetylene, the branching steps may also release significant heat [27]. Three-step models [25, 28, 26, 29] allow much more flexibility than single-step kinetics which unavoidably associates heat release with initiation, and offer additional physical insight with a rather negligible increase in computational cost.

The reaction steps are represented by

$$
\begin{array}{ll}
\text { Initiation } & \mathcal{F} \stackrel{k_{I}}{\rightarrow} \mathcal{R} \\
\text { Branching } & \mathcal{F}+\mathcal{R} \stackrel{k_{B}}{\longrightarrow} 2 \mathcal{R} \\
\text { Termination } & \mathcal{R} \stackrel{k_{T}}{\longrightarrow} \mathcal{P}
\end{array}
$$

The species equations and net production/consumption rates of fuel, $\dot{\omega}_{\mathcal{F}}$, and chain-carriers, $\dot{\omega}_{\mathcal{R}}$, based on the scheme above are: 


$$
\begin{aligned}
& \frac{\partial \rho Y_{\mathcal{F}}}{\partial t}+\frac{\partial \rho u_{i} Y_{\mathcal{F}}}{\partial x_{i}}=\dot{\omega}_{\mathcal{F}}=-r_{I}-r_{B} \\
& \frac{\partial \rho Y_{\mathcal{R}}}{\partial t}+\frac{\partial \rho u_{i} Y_{\mathcal{R}}}{\partial x_{i}}=\dot{\omega}_{\mathcal{R}}=r_{I}+r_{B}-r_{T}
\end{aligned}
$$

with

$$
\begin{aligned}
& r_{I}=k_{I}\left(\rho Y_{\mathcal{F}}\right) \exp \left(-E_{I} / R_{u} T\right) \\
& r_{B}=k_{B}\left(\rho Y_{\mathcal{F}}\right)\left(\rho Y_{\mathcal{R}}\right) / W \exp \left(-E_{B} / R_{u} T\right) \\
& r_{T}=k_{C}\left(\rho Y_{\mathcal{R}}\right)
\end{aligned}
$$

The corresponding pre-exponential reaction constants for initiation, branching and termination are $k_{I}=k_{C} \exp \left(E_{I} / R_{u} T_{I}\right), k_{B}=k_{C}(W / \rho) \exp \left(E_{B} / R_{u} T_{B}\right)$, and $k_{T}=k_{C}$, respectively. $E_{I} / R_{u}$, and $E_{B} / R_{u}$ are the initiation and branching activation temperatures, and $T_{I}$ and $T_{B}$ are the crossover temperatures at which the chain-initiation and branching rates become equal to the chain-termination rate. Finally, the expression for $e_{s}$ is the same as for the single-step model, and the local heat release is given by $q=-\left(1-Y_{\mathcal{F}}-Y_{\mathcal{R}}\right) Q$.

\subsubsection{Detailed kinetics}

The detailed mechanism of Mével for the oxidation of hydrogen was used [30], which includes 9 species and 21 reactions. This model has been extensively validated against experimental databases for laminar burning speeds, ignition delay times and detonation relevant conditions [31]. For detailed kinetics, the sensible internal energy and local heat release are given by $e_{s}=\int_{T_{\mathrm{ref}}}^{T} c_{v} d T$, and $q=-\sum_{k=1}^{N} \dot{\omega}_{k} \Delta h_{f, k}^{o} ; c_{v}$ and $\Delta h_{f, k}^{o}$ are the specific heat at constant volume of the mixture and the entalphy of formation of species $k$. JANAF polynomials are 
used to account for temperature dependent thermodynamics, i.e. specific heats, internal energy and enthalpy of the mixture.

\subsubsection{Determination of model parameters for simplified kinetics}

To model the behavior of a stoichiometric $\mathrm{H}_{2}-\mathrm{O}_{2}$ mixture, the kinetic parameters were determined, for single-step chemistry, by varying $A_{s}$ and $E_{a} / R_{u}$ to match the constant volume ignition delay times, $\tau_{\text {ind }}$, obtained using the detailed mechanism of Mével et al. [30]. For three-step chain branching chemistry, the same methodology was used to tune $k_{C}, E_{I} / R_{u}, E_{B} / R_{u}, T_{I}$ and $T_{B}$. The calibration comprises two steps: first, $\tau_{\text {ind }}$ at the von Neumann state $\left(T_{\mathrm{vN}}, p_{\mathrm{vN}}, \rho_{\mathrm{vN}}\right)$ was targeted; the kinetic parameters were varied to match it to within $0.1 \%$. Second, at fixed $\rho_{\mathrm{vN}}$, the process was repeated for $900 \mathrm{~K} \leq T \leq 2000 \mathrm{~K}(1.6 \mathrm{MPa} \leq p \leq 3.6$ $\mathrm{MPa}$ ). For single-step chemistry, $A_{s}$ and $E_{a} / R_{u}$ respectively control the $\tau_{\text {ind }}$ value and the rate at which it decreases/increases as a function of $T$ (i.e. the slope). For the three-step chain-branching, $E_{I} / R_{u}$ controls the slope for the high temperature range, whereas $E_{B} / R_{u}$ does it for the low temperature range. $k_{C}$, shifts $\tau_{\text {ind }}$ to higher/lower values, and $T_{B}$ is the chain-branching cross-over temperature (location where the change of slope in Fig. 1 is observed). This calibration procedure yields a good compromise with regards to matching both, the high and low temperature ranges, with the available degrees of freedom. Special attention was paid to the high temperature range, and particularly to the von Neumann state.

The parameters found are $A_{s}=6.0 \times 10^{9} \mathrm{~s}^{-1}$, and $E_{a} / R_{u}=14160 \mathrm{~K}$, for single step; and $k_{C}=2 \times 10^{7} \mathrm{~s}^{-1}, E_{I} / R_{u}=25000 \mathrm{~K}, E_{B} / R_{u}=8500 \mathrm{~K}$, $T_{I}=2431 \mathrm{~K}, T_{B}=1350 \mathrm{~K}$, for three-step chain-branching chemistry. A constant molecular weight of $W=12 \mathrm{~g} / \mathrm{mol}$, and ratio of specific heats of $\gamma=1.33$, are used; $Q=4.80 \mathrm{MJ} / \mathrm{kg}$ and $4.99 \mathrm{MJ} / \mathrm{kg}$ for single-step and three-step chain- 
branching chemistry, respectively.
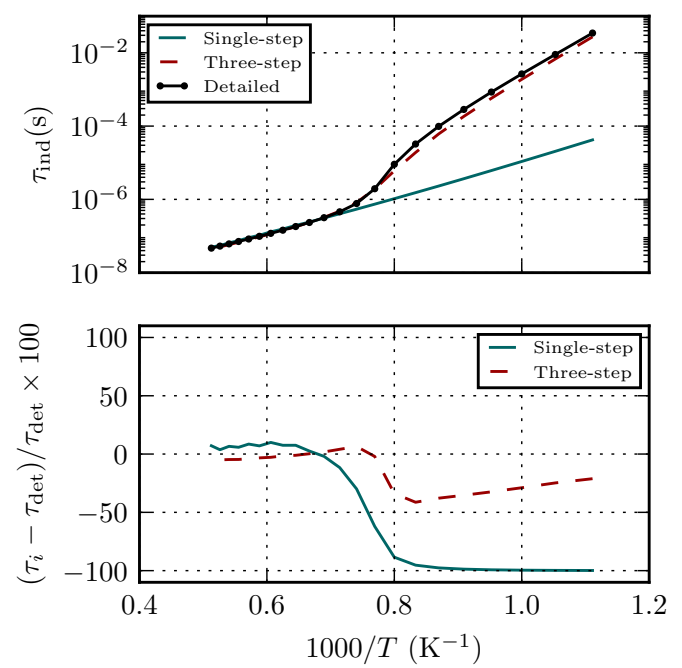

Figure 1: Top: constant volume induction times as a function of inverse temperature. Initial conditions (von Neumann state $-\rho_{\mathrm{vN}}$ ) computed for a stoichiometric $\mathrm{H}_{2}-\mathrm{O}_{2}$ mixture at $p_{o}=100$ $\mathrm{kPa}$ and $T_{o}=295 \mathrm{~K}$. At fixed $\rho_{\mathrm{vN}}$, temperature was varied in the range $900 \mathrm{~K} \leq T \leq 2000 \mathrm{~K}$, resulting in associated pressure variations of 1.6 - 3.6 MPa. Bottom: percent deviation for singlestep and three-step chain-branching with respect to detailed chemistry.

Figure 1 shows the ignition delay times predicted by each of the three chemical models, and the percent deviation computed for single-step and three-step chain-branching chemistry using the ignition delay times from detailed chemistry, $\tau_{\text {det }}$, as a reference. Both kinetic models predict approximately the same ignition delay time as detailed chemistry in the high temperature range $(T \geq 1429 \mathrm{~K})$ with average deviations of less than $10 \%$ and $5 \%$ for single-step and three-step chainbranching chemistry, respectively. The change of activation energy typical of $\mathrm{H}_{2}$ chemistry, i.e. change of slope in black line, is expectedly not captured by the single-step model. The three-step on the other hand predicts the low temperature 
delay times with average deviations of less than $20 \%$, and shows a maximum deviation of less than $50 \%$ at $T \sim 1250 \mathrm{~K}$. For lower temperatures $(T<1250 \mathrm{~K})$, the differences in ignition delay times increase from one to three orders of magnitude for single-step chemistry.

\subsection{Numerical methods}

The governing equations were integrated using our parallel in-house code RESIDENT (REcycling mesh SImulation of DEtonations). Spatial discretization is performed using finite differences together with directional splitting; operator splitting couples the hydrodynamics and the chemistry. The characteristic variables are reconstructed at the cell boundaries using a ninth order monotonicity preserving scheme [32], which allows for a reduction of dissipation and provides improved accuracy even in the presence of strong flow discontinuities, i.e shocks and contact surfaces,[33,34]. The Approximate Riemann solver of Toro (HLLC) [35] with the fix of Shen et al. [36] for the shear wave is used to compute the numerical fluxes. A third-order TVD explicit Runge-Kutta is selected for the temporal integration with a CFL number of 0.2. Additionally, a sliding window technique was used to follow the detonation as it propagated into mixture at rest. This allowed us to have a fixed number of cells in the computational domain at all times, irrespective of the length of the channel computed $[37,38]$. Special care was taken to ensure that the size of the window used did not influence the flow structure and quenching limits reported.

The chemistry is integrated as follows: using an analytical solution that entails freezing the Arrhenius term during the chemical time step for single-step; explicitly for three-step chain-branching; and using a semi-implicit preconditioned iterative method based on a diagonal approximation of the chemical Jacobian for 
detailed chemistry, (see Savard et al. [39]). This numerical integration method has been validated for stiff chemistry, and shown to be stable and to provide good convergence rates for time steps larger than $1 \mu \mathrm{s}$. Note that this is useful in the context of direct numerical simulation of turbulent flames, where low Mach number approximations are the norm, and time steps are often restricted by the chemistry instead of the convective time scale. For high speed compressible flow however, the convective and chemical time scales are comparable and typical time steps are significantly lower than $1 \mu \mathrm{s}$, hence the species lifetimes contained in the diagonal preconditioner will necessarily capture all critical small chemical time scales. In our calculations, only one iteration step is required.

\subsection{Domain, initial and boundary conditions}

A schematic of the simulation setup is shown in Fig. 2. The simulations were run in two steps. First, a detonation was initiated and allowed to propagate in a channel completely filled with reactive mixture, i.e. stoichiometric $\mathrm{H}_{2}-\mathrm{O}_{2}$, until a quasi-steady structure was achieved ( $\sim 100 \mu$ s of propagation). The initial conditions used were $p_{o}=100 \mathrm{kPa}, T_{o}=300 \mathrm{~K}$ with mass fractions of fuel, $Y_{\mathcal{F}, o}$ $=1$, for single-step kinetics; and fuel and radical species $Y_{\mathcal{F}, o}=1$ and $Y_{\mathcal{R}, o}=0$, for three-step chain-branching kinetics. For detailed chemistry, the initial mass fractions of $\mathrm{H}_{2}$ and $\mathrm{O}_{2}$ are $Y_{\mathrm{H}_{2}, o}=0.1112$ and $Y_{\mathrm{O}_{2}, o}=0.8888$.

Second, the resulting fields were used as initial conditions for separate simulations in which the channel height was filled with a layer of inert mixture. In order to have a meaningful comparison with the simplified models the acoustic impedance ratio, $(\rho a)_{\text {inert }} /(\rho a)_{\text {react }}$ where $a$ is the speed of sound, was kept constant to a value representative of stoichiometric $\mathrm{H}_{2}-\mathrm{O}_{2}$ bounded by $\mathrm{N}_{2}\left(Y_{\mathrm{N}_{2}, o}=1\right)$ at $T_{o},\left[\left(\gamma_{\text {inert }} W_{\text {inert }} T_{\text {react }}\right) /\left(\gamma_{\text {react }} W_{\text {react }} T_{\text {inert }}\right)\right]^{1 / 2}=1.52$ 

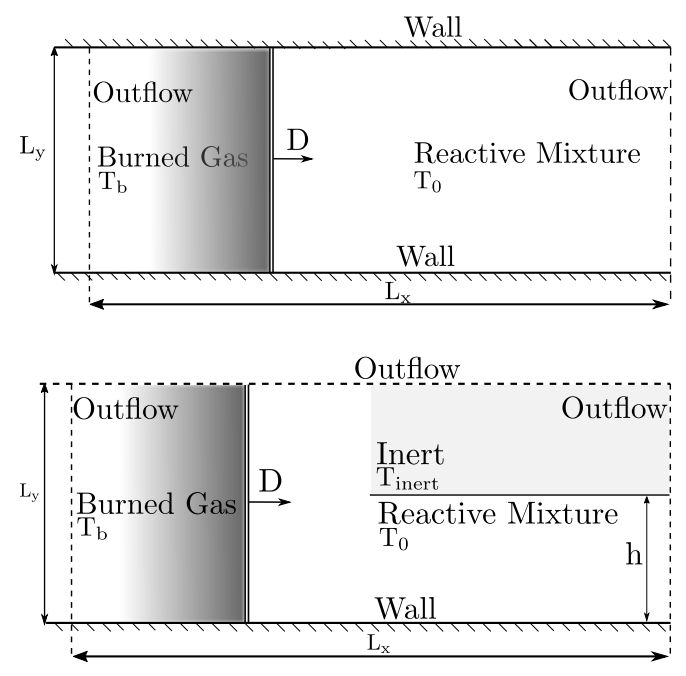

Figure 2: Simulation setup and computational strategy to determine the critical height.

. Given the assumptions of the simplified kinetics, i.e. constant $\gamma$ and $W$, the temperature of the inert layer was computed accordingly, therefore characterized by $Y_{\mathcal{F}, o}=Y_{\mathcal{R}, o}=0$, and $T_{\text {inert }}=130 \mathrm{~K}$. The boundary conditions are specified in Fig. 2.

Different reactive layer heights, $h$, were tested to determine the height at which successful detonation propagation is no longer possible, $h_{\text {crit }}$. The resolution used was uniform and set to $\Delta x=\Delta y=l_{\text {ind }} / 10$ pts. A detailed numerical convergence study, included in Appendix A, was carried out to ensure the resolution chosen was sufficient. The computations were performed using $\sim 400-500$ processsors with an average of 70 million cells per case; the full study took 2.5 million CPU hours. 


\section{Results}

\subsection{Ideal detonation}

The simulations run in uniform mixture allow to highlight the differences in global features among the chemical models. Therefore, in this subsection, we compare their detonation front dynamics, instantaneous flow fields and numerical soot foils.

\subsubsection{Detonation front dynamics}
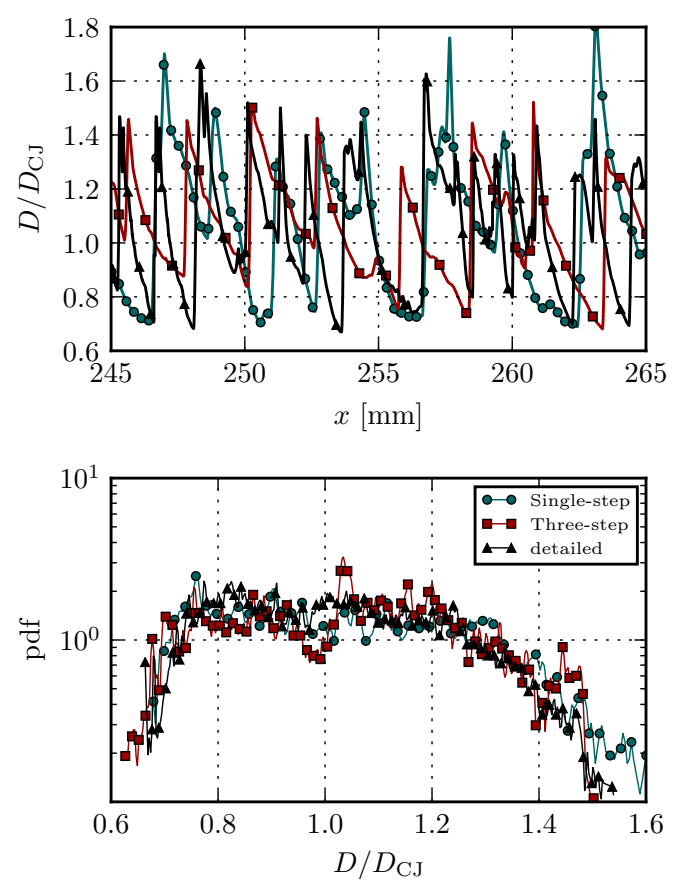

Figure 3: Normalized instantaneous shock front velocity as a function of distance (top), and probability density function of the leading shock velocity (down) for single-step, three-step chainbranching and detailed chemistry - ideal detonation. The detonation has propagated about 100 $\mu \mathrm{s}$. 
Figure 3 shows the normalized instantaneous detonation velocity, $D / D_{\mathrm{CJ}}$, and its probability density function (pdf) measured at the bottom wall of the channel. The velocity peaks arise from transverse wave and triple point collisions. The latter collisions give rise to local Mach stems that subsequently decay and evolve to an incident shock as the speed of the front drops below $D_{\mathrm{CJ}}$, inducing a local decoupling with the reaction zone. A new cycle begins, upon new triple point collisions, resulting in an abrupt acceleration of the front. This behavior is characteristic of detonations and all chemical models capture it.

Several features stand out: (i) all chemical models show similar dynamics regarding the rates at which their pdf's decay for $D / D_{\mathrm{CJ}}<0.8$ and $D / D_{\mathrm{CJ}} \geq 1.2$, as well as the values attained in the range $0.8<D / D_{\mathrm{CJ}}<1.2$ exhibiting a rather flat region. This behavior seems to differ from what previous researchers have reported experimentally [40], and numerically [41], however the conditions here are for larger domains that include many cells across the channel's height. (ii) Differences are only observed for $D / D_{\mathrm{CJ}}$ values around unity, and $D / D_{\mathrm{CJ}} \sim 1.4$. The pdf for three-step chain-branching lies above and below that of detailed and single-step chemistry, respectively.

Finally, note that when $D / D_{\mathrm{CJ}}<0.86$ for thee-step chain-branching chemistry, the post-shock temperature is below the crossover temperature $\left(T_{\mathrm{s}}<T_{B}\right)$ hence the production of active intermediates is slow resulting in a significant increase in $\tau_{\text {ind }}$ with values that are about two orders of magnitude longer than those associated with constant volume ignition behind detonation fronts $(\sim 1 \mu \mathrm{s})$. This will be shown to affect the nature of the flow fields. Overall the front dynamics 
seems to be reasonably captured by both simplified kinetics schemes.

\subsubsection{Instantaneous structure}

Instantaneous two-dimensional fields of normalized temperature $\left(T / T_{o}\right)$, and magnitude of the density gradient $\left(|\nabla \rho| /\left|\nabla \rho_{\max }\right|\right)$ are displayed in Fig. 4 for the three models. The fields are taken after $\sim 100 \mu$ s corresponding to $\sim 300 \mathrm{~mm}$ of propagation. This guarantees that a quasi-steady structure, independent of the initiation transient, was achieved. Mach stems and incident shocks are linked with transverse waves which propagate back and forth along the detonation front, becoming weaker as they get away from the front. Unburnt pockets result from their collisions

Three different physical mechanisms contribute to the consumption of these pockets: (i) constant volume explosion enhanced by transverse waves that repeatedly change their thermodynamic state; (ii) transverse waves; (iii) mixing and diffusive burning. The former two are the only ones the inviscid model used in this work would capture. In the normalized density gradient fields for detailed chemistry, smaller flow structures and seemingly stronger transverse waves than those observed for single-step and three-step chain-branching chemistry are present. This is in line with the observations of Taylor et al. [42]. Finally, the unburned pockets are larger and seem to last longer downstream of the leading shock for both simplified models considered, whereas detailed chemistry shows a more uniform burned gas temperature downstream of the front. The changes in induction length, $l_{\text {ind }}$, along the height of the front for all chemical models, as seen from these fields, seem to have comparable sizes. 

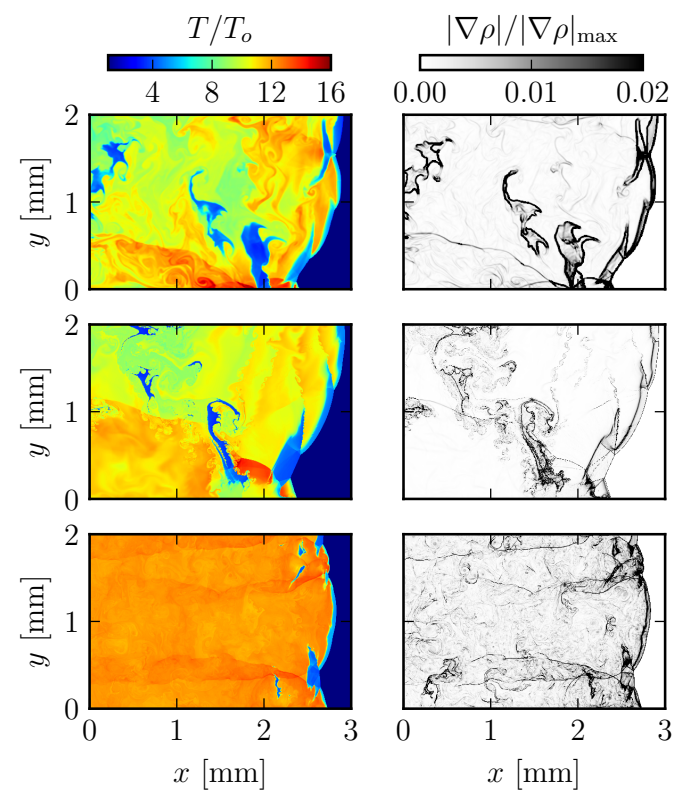

Figure 4: Instantaneous fields for the three chemical models considered. Top: single-step; center: three-step chain-branching; bottom: detailed chemistry. 


\subsubsection{Numerical soot-foils}

Numerical soot foils were obtained by tracking the pressure maxima per cell during the simulation. These records were started after the detonation completed the initial transient after initiation. Despite the differences observed in the twodimensional instantaneous fields, all chemical models yield similar features regarding the detonation cell size irregularity (see Fig. 5). Histograms showing the distribution of cell sizes are also included in Fig. 5. These were obtained by manually measuring all the length scales present (90 samples per case), and subsequently sorting the data by number of occurrences of a given scale, i.e. Frequency (\#). This analysis allowed us to define a characteristic cell size for each chemical model as well as to more accurately determine their average. The simplified kinetics schemes show a more broad distribution of length scales than that of detailed chemistry, which also seems to be confined to the lower end of the spectrum. The average cell sizes are $1.7,1.3$ and $0.8 \mathrm{~mm}$ for single-step, threestep chain-branching and detailed chemistry, respectively. Their characteristic cell size based on the histograms are 1.8, 1.2 and $0.6 \mathrm{~mm}$. The cell size was thus numerically estimated to lie in the range of $0.8 \mathrm{~mm} \leq \lambda_{\text {num }} \leq 1.7 \mathrm{~mm}$ whereas the cell size reported experimentally is in the range of $1.4 \mathrm{~mm} \leq \lambda_{\exp } \leq 2.1 \mathrm{~mm}$ [43]. The smaller cell size predicted by detailed chemistry is somewhat expected and not due to lack of resolution (see Appendix A) but rather due to potential vibrational non-equilibrium effects $[42,44]$, reaction model uncertainties and/or threedimensional effects [45].

\subsection{Semi-confined detonation}

The differences between the three models in the semi-confined case are investigated in this subsection. The quasi-steady detonation computed above will 

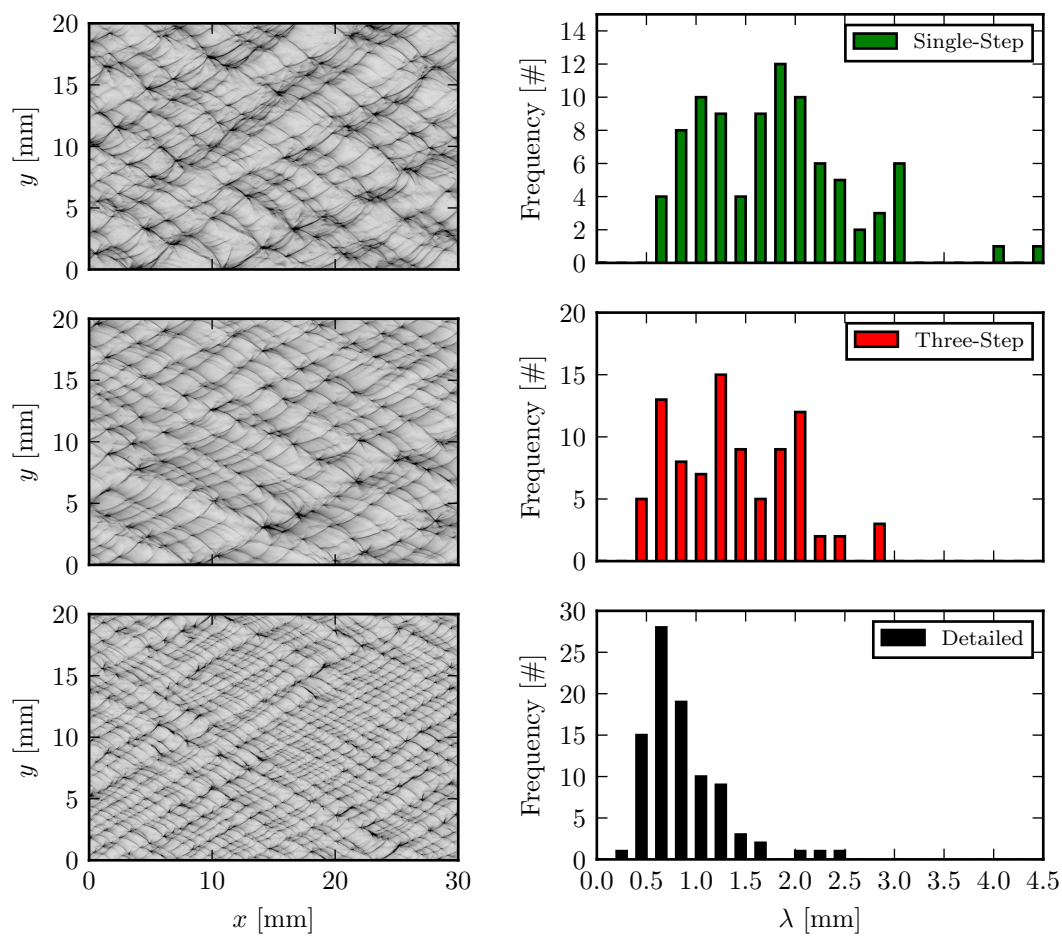

Figure 5: Numerical soot-foils and histograms showing the distribution of cell sizes $(\lambda)$ for singlestep (top), three-step chain-branching (center) and detailed chemistry (bottom). The height of the reactive layer is $h=20 \mathrm{~mm}$.

now propagate into reactive mixture bounded by an inert layer. Based on previous work performed in our group [11] with single-step chemistry fitted to match the numerically determined cell size and steady detonation velocity with those reported experimentally [43], the critical height for a stoichiometric $\mathrm{H}_{2}-\mathrm{O}_{2}$ mixture bounded by $\mathrm{N}_{2}$ was found to be $h_{\text {crit }}=18 \mathrm{~mm}$. However, since $E_{a} / R_{u}$ is higher for the current fitting in which we take $\tau_{\text {ind }}$ as a target, $h_{\text {crit }}$ is expected to increase $[10,11,13]$. Using a layer height slightly above this value, $h=20 \mathrm{~mm}$, we carried out simulations to compare the main features among the three chemical 
models.

\subsubsection{Global features}

Figure 6 shows a comparison of the flowfields obtained after $20 \mu$ s of propagation. In all cases, characteristic traits of detonations propagating under yielding confinement are recovered, namely a curved front, an oblique transmitted shock and a shear layer separating shocked inert gas from detonation products. However, significant differences appear. While the detonation seems to propagate comfortably in the detailed chemistry case, the structure in the single-step and three-step cases exhibits a more irregular behavior with sizable pockets of unburnt gas distributed over the flow field, as well as larger distances between the leading shock and the reaction zone in the vicinity of the interface. The detonation front is curved for all chemical models which is an inherent signature of a velocity deficit. Detailed chemistry seems to be less affected by the inert boundary showing less curvature, whereas single-step and three-step chemistry shows larger bulbs and more pronounced cusps.

Further examination of the flow field at longer times (Fig. 7), after $\sim 100 \mu \mathrm{s}$ of interaction with the interface, reveals that the detonation continues to propagate without any issues for detailed chemistry; single-step and three-step chainbranching chemistry, on the other hand, are incapable of sustaining a detonation at this layer height. This outcome suggests that the quenching limits, characterized here using $h_{\text {crit }}$, are dependent on the choice of chemical modeling used. We emphasize however, that alternative calibration methods using fitting targets other than $\tau_{\text {det }}$ could lead to different results. In order to find $h_{\text {crit }}$, the value of $h$ should be increased for single-step and three-step chain-branching, and decreased for detailed chemistry. Since for the former two cases the detonation failed to 

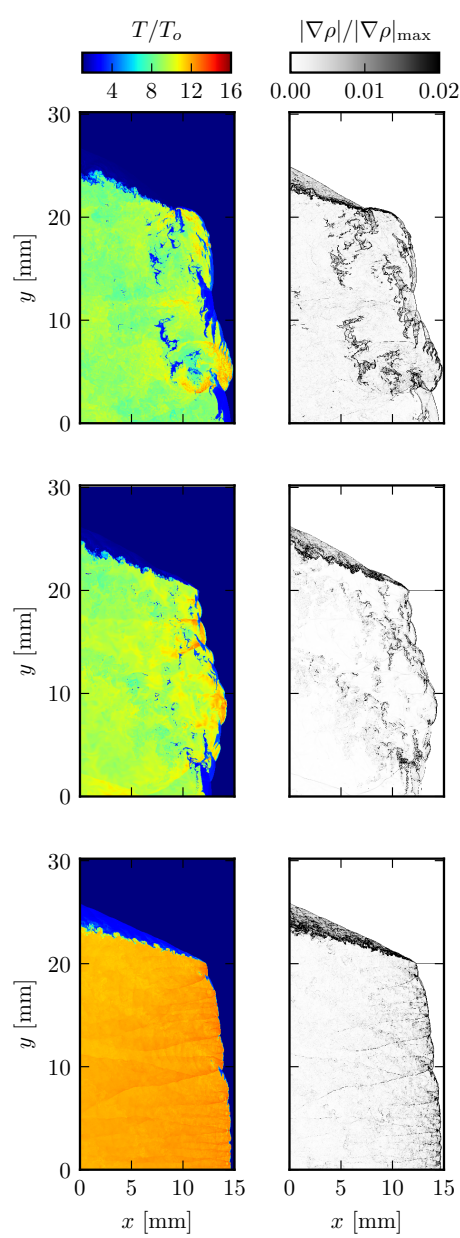

Figure 6: Instantaneous detonation structure for single-step (top), three-step chain-branching (center) and detailed chemistry (bottom) after $20 \mu \mathrm{s}$ of propagation into the reactive/inert layer complex. The height of the reactive layer is $h=20 \mathrm{~mm}$. The solid horizontal line in normalized magnitude of density is a visual aid showing the location of the reactive/inert interface.

propagate, whereas for the latter it transmitted successfully. Fifteen additional simulations were run, progressively increasing and decreasing the value of $h$, to quantify the differences in $h_{\text {crit }}$ among the chemical models. 

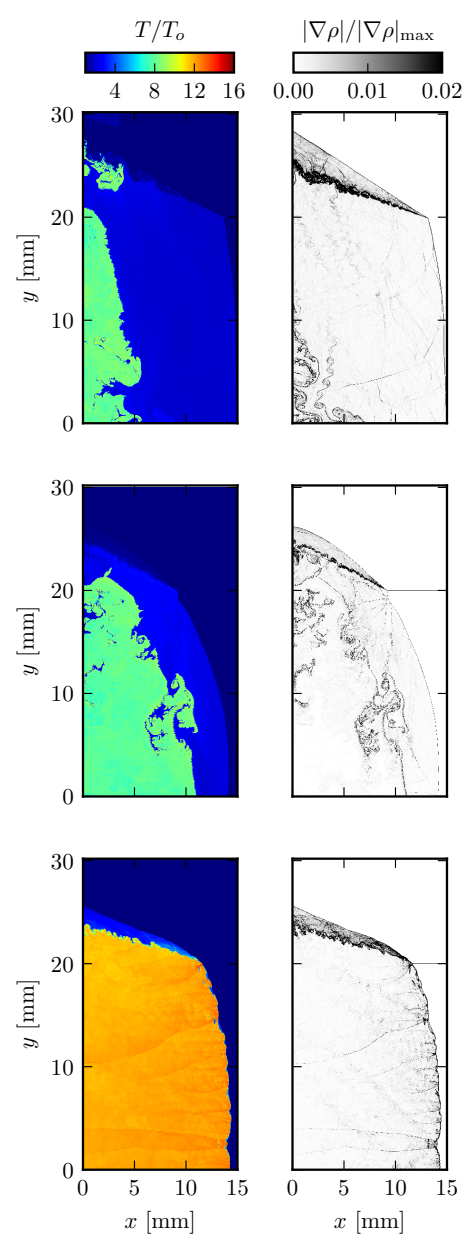

Figure 7: Instantaneous detonation structure for single-step (top), three-step chain-branching (center) and detailed chemistry (bottom) after $100 \mu \mathrm{s}$ of propagation into the reactive/inert layer complex. The height of the reactive layer is $h=20 \mathrm{~mm}$. The solid horizontal line in normalized magnitude of density is a visual aid showing the location of the reactive/inert interface.

\subsubsection{Critical heights and quenching dynamics}

The values of $h_{\text {crit }}$ found are 24, 20 and $6 \mathrm{~mm}$ for single-step, three-step and detailed chemistry, respectively. While the differences in flow structure were more 
pronounced when comparing the models at fixed heights (Figs. 6 and 7), close to $h_{\text {crit, }}$, the flow fields show some similarities (Fig. 8), namely increased curvature and presence of unburnt pockets, as well as two distinct zones: (i) decoupling of the leading shock and reaction zone close to the interface; and (ii) nearly undisturbed propagation as the bottom wall is approached.

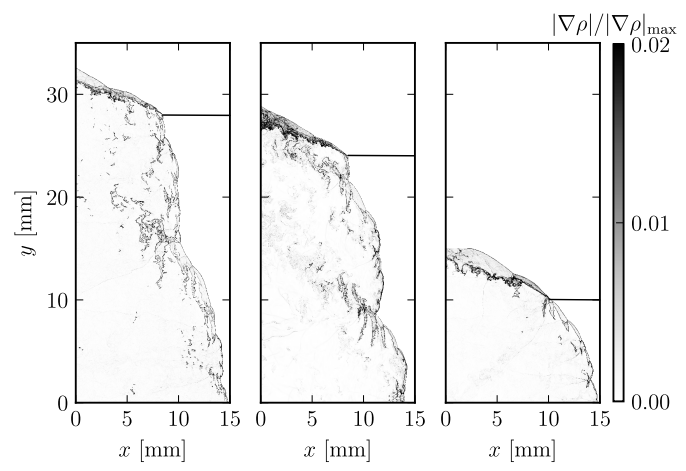

Figure 8: Comparison of flow structures at heights slightly above $h_{\text {crit }}$ for single-step (left - $h=28$ $\mathrm{mm}$ ), three-step chain-branching (center - $h=24 \mathrm{~mm}$ ) and detailed chemistry (right $-h=10$ $\mathrm{mm})$.

To further investigate the quenching dynamics, numerical soot foils for heights slightly above $h_{\text {crit }}$ were examined to observe how the detonation wave experiences losses. The reactive-inert layer complex is located $10 \mathrm{~mm}$ away from the left boundary $(x=10 \mathrm{~mm})$ for all the chemical models tested.

Figure 9 displays soot foil records with single-step for $h=28 \mathrm{~mm}$ (top) and $h_{\text {crit }}=24 \mathrm{~mm}$ (bottom). For $h>h_{\text {crit }}$, the detonation successfully propagates, the initial interaction with the interface results in oblique streaks traveling toward the bottom wall along which the cell size increases. This increase in cell size is caused by unsteady expansion waves coming from the interface which partially decouple the leading shock and reaction zone. Upon reflection at the bottom wall 
( $x \sim 100 \mathrm{~mm}$ ) a new curved streak of cells is formed, now oriented towards the interface ( $x=140-150 \mathrm{~mm}$ ); upstream of it, re-ignition occurs accompanied by smaller cells suggesting the presence of a locally overdriven front that subsequently propagates without failing through the reactive layer. For $h=h_{\text {crit }}$, the dynamics is similar to that described above, except that after the first reflection from the bottom wall, the detonation is not able to continue propagating. The thermodynamic state $(p, T)$ generated at the wall upon reflection, and the chemical time scale associated with it, is not strong enough to compete with the expansion induced cooling coming from the interface which results in total quenching of the front. The distance to quenching, $x_{\mathrm{Q}}$, defined as the first location where full decoupling is observed after interaction with the interface, lies around $200 \mathrm{~mm}$ for single-step chemistry.

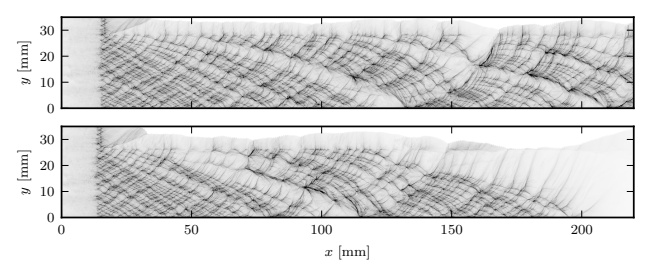

Figure 9: Numerical soot foils for single-step chemistry for two reactive layer heights: $h=28 \mathrm{~mm}$ - top; $h_{\text {crit }}=24 \mathrm{~mm}$ - bottom.

The numerical soot foils for three-step chain-branching kinetics show a somewhat different evolution for $h=24 \mathrm{~mm}>h_{\text {crit }}$ (Fig. 10 - top). Two phases in the transients of the interaction are evident: (i) a thick clear band forms immediately after contact with the interface between inert and reactive mixture $(10 \mathrm{~mm}<x<$ $75 \mathrm{~mm}$ ), the absence of cells in this region indicate that full decoupling between the reaction zone and leading shock occurred leading to partial quenching of the 
front. This is in contrast with the dynamics described above for single-step kinetics where only a gradual increase of cell size was observed. (ii) Upon reflection at $x \sim 90 \mathrm{~mm}$, and following the interaction with ignition centers generated at the interface that sweep inward toward the bottom wall, the front is fully re-initiated at $x \sim 98 \mathrm{~mm}$. Similar observations were reported in experiments performed by Murray and Lee [19]. For $h=h_{\text {crit }}=20 \mathrm{~mm}$, Fig. 10 - bottom, shows essentially the same quenching mechanism as that described for single-step kinetics, with seemingly stronger expansion waves entering the reaction zone leading to full decoupling and failed re-ignition following triple point reflections from the bottom wall at $x=120 \mathrm{~mm} ; x_{\mathrm{Q}}=140 \mathrm{~mm}$ for three-step chain-branching chemistry.

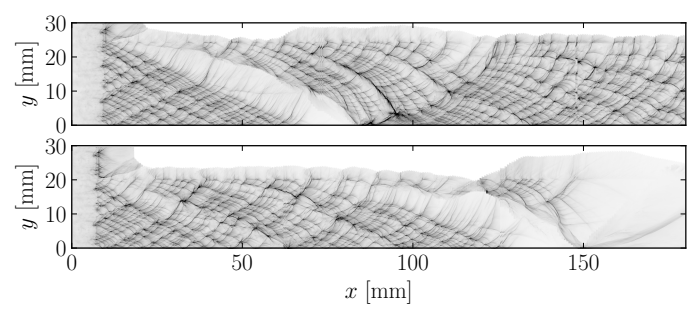

Figure 10: Numerical soot foils for three-step chain-branching chemistry for two reactive layer heights: $h=24 \mathrm{~mm}$ - top; $h_{\text {crit }}=20 \mathrm{~mm}$ - bottom.

The evolution obtained for detailed chemistry seems to be more rich including some of the features observed in single-step and three-step chain-branching chemistry. For $h=10 \mathrm{~mm}>h_{\text {crit }}$ (Fig. 11 - top) shortly after the interaction with the interface $(10 \mathrm{~mm}<x<55 \mathrm{~mm})$, the cell size increases gradually without local quenching in line with the dynamics described for single-step chemistry; for $50 \mathrm{~mm}<x<115 \mathrm{~mm}$ the thick clear band is evidence of full local quenching of the front, now in line with the dynamics described for three-step chain-branching chemistry. In contrast with the two previous cases, re-establishment of the det- 
onation wave takes place through several quenching and re-ignition steps. Final successful re-ignition occurs upon reflection from the bottom wall at $x \sim 105 \mathrm{~mm}$; ignition centers emerging from the yielding confinement re-initiate the detonation wave $[17,18]$. For $h=h_{\text {crit }}=6 \mathrm{~mm}$ (Fig. 11 - bottom), the failure of the front occurs through a gradual separation between the reaction zone and leading shock as cooling induced expansion emanating from the interface quenches the chemistry. No local quenching or re-ignition attempts were observed at this reactive layer height. $x_{\mathrm{Q}}$, for detailed chemistry was $60 \mathrm{~mm}$. Three-step chain-branching and detailed chemistry show similar re-initiation dynamics.

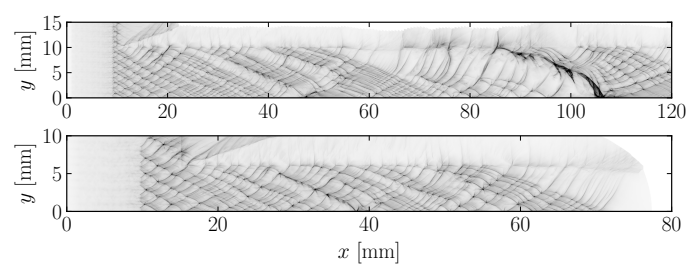

Figure 11: Numerical soot foils for detailed chemistry for two reactive layer heights: $h=10 \mathrm{~mm}$ - top; $h_{\text {crit }}=6 \mathrm{~mm}$ - bottom. Note that the horizontal and vertical scales are different to ease visualization of the cellular structure.

Table 1 summarizes the critical heights and quenching distances obtained. The value of $h_{\text {crit }}$ predicted by detailed chemistry is in very good agreement with experimentally reported values [22]. Simplified kinetics yield $h_{\text {crit }}$ values that are more than three times (single-step), and four times (three-step) larger than those obtained with detailed chemistry. Finally, the quenching distances, $x_{\mathrm{Q}}$, increase with decreasing chemical modeling complexity as $x_{\mathrm{Q} \text {, Detailed }} \ll x_{\mathrm{Q}, 3-\text { Step }}<x_{\mathrm{Q}, 1-\text { Step }}$. 
Table 1: Critical heights, $h_{\text {crit }}$, and quenching distances, $x_{Q}$, predicted with different chemical models.

\begin{tabular}{ccc}
\hline Chemistry modeling & $h_{\text {crit }}(\mathrm{mm})$ & $x_{Q}(\mathrm{~mm})$ \\
\hline Single-step & 24 & 200 \\
Three-step & 20 & 140 \\
Detailed & 6 & 60 \\
Experimental [18] & 4.6 & - \\
\hline
\end{tabular}

\subsubsection{Unburnt pocket analysis}

In an attempt to understand the increased resistance that detailed chemistry exhibits we analyze in detail one of the mechanisms whereby unburnt pockets get consumed, i.e. constant volume explosion, and also provide a simple estimate of the time scales for diffusive burning. Figure 12 shows an overview of the full flow field shortly after interaction with the inert interface, and a zoom to the region of interest.

Using the thermodynamic state ( $p$ and $T$ ) in five different locations inside the unburnt pocket (see close-up to normalized density gradient) constant volume delay times were computed in two different ways: (i) assuming the composition to be that of fresh mixture $\left(Y_{\mathrm{H}_{2}, o}\right.$ and $\left.Y_{\mathrm{O}_{2}, o}\right)$ - case 1; (ii) using the actual local compositions $\left(Y_{i}\right)$ - case 2. This was done to assess the effect of incipient intermediates on the delay times. For single-step and three-step chemistry, only case 1 can be considered using $Y_{f}=1$.

The presence of intermediates expectedly resulted in faster $\tau_{\text {ind }}$ (see Fig. 13) but the differences are not significant to provide any clue regarding the increased 

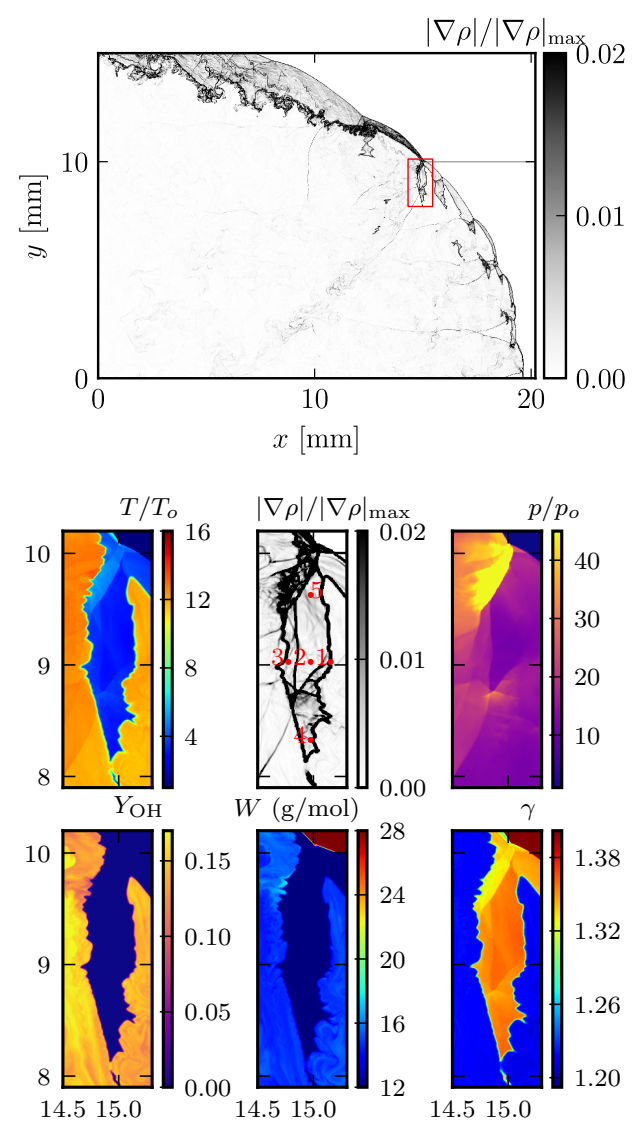

Figure 12: Top: normalized magnitude of density gradient, $\nabla \rho / \nabla \rho_{\max }$, of full flow field. Bottom: close-up to unburnt pocket on one pocket of unburnt gas.

resistance of the wave to failure. Bear in mind however that the thermodynamic state of this pocket is constantly changing as it is subjected to transverse and expansion waves of various strengths whose overall effect could result in an effective decrease in $\tau_{\text {ind }}$. Moreover, there are variations of more than one order of magnitude in $\tau_{\text {ind }}$ across the points examined that single-step chemistry does not capture.

A simple order of magnitude estimate to determine whether the time scales for diffusive burning are comparable with those computed above for the chemistry, is 


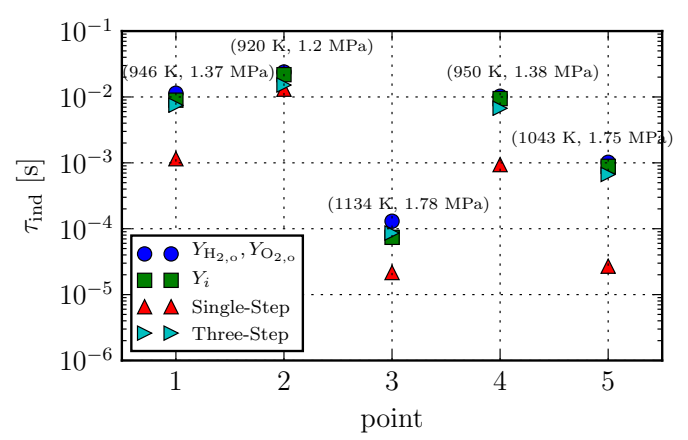

Figure 13: Constant volume delay times, $\tau_{\text {ind }}$, computed at different locations inside the unburnt pocket.

to take the ratio of the square of a characteristic length scale associated with the pocket $(0.1-0.5 \mathrm{~mm}$ - measured from the flow field $)$ to the thermal diffusivity of the mixture at the local thermodynamic conditions, $\tau_{\text {diff }}=L^{2} / \alpha$. Taking the minimum and maximum values of $p$ and $T$ in the pocket $(1205 \mathrm{kPa} ; 920 \mathrm{~K}$ and $1782 \mathrm{kPa} ; 1134 \mathrm{~K}), \alpha$ varies from $4.317 \times 10^{-5}$ to $4.536 \times 10^{-5} \mathrm{~m}^{2} / \mathrm{s}$. Taking its arithmetic average and the values of $L$ above, the diffusive time scale ranges between $2.226 \times 10^{-4} \mathrm{~s} \leq \tau_{\text {diff }} \leq 5.648 \times 10^{-3} \mathrm{~s}$ which are of the same order of $\tau_{\text {ind }}$ in Fig. 13 but far from typical detonation time scales $(\sim 1 \mu \mathrm{s})$. Based on this estimate it is plausible that diffusive effects may play a role in burning the pockets but since the inviscid model used here does not include them and the value of $h_{\text {crit }}$ obtained is in agreement with experimental observations, there must be other factors at play present in detailed chemistry driving the dynamics of quenching.

Detailed chemistry accounts for changes in molecular weight, $W$, and ratio of specific heats, $\gamma$, as a function of $T$ typically neglected in simplified descriptions of the chemistry, as well as for the various chemical time scales associated with each of the elementary reactions present in the mechanism. Fig.12 displays the 
variations in $W$ and $\gamma$ within the unburnt pocket, as it transits to burnt conditions. $W$ varies from $12 \mathrm{~g} / \mathrm{mol}$ in shocked fresh mixture to $14-16 \mathrm{~g} / \mathrm{mol}$ in burnt mixture (due to dissociation) leading to an increase in temperature of the products, whereas $\gamma$ shows more significant variations within the pocket itself $(1.33-1.36)$ followed by a decrease toward $\sim 1.21$ in burnt products. None of these observations allow to provide conclusive answers regarding the differences in $h_{\text {crit }}$ obtained among the chemical models.

\subsubsection{Comparison among the ZND profiles}

The ZND (Zel'dovich-von Neumann-Döring) structure obtained with all chemical models is shown in Fig. 14. The characteristic lengths for each are: a half reaction length of $l_{1 / 2,1 \text {-Step }}=70 \mu \mathrm{m}$ for single-step, defined as the distance from the leading shock to the location where half of the fuel is consumed. As mentioned earlier, single-step models do not have a clearly defined thermally neutral zone before heat release; for three-step chain-branching and detailed chemistry: $l_{\text {ind, 3-Step }}=17 \mu \mathrm{m}$ and $l_{\text {ind, Detailed }}=35 \mu \mathrm{m}$, respectively, formally defined as the distance from the leading shock to maximum thermicity $\dot{\sigma}_{\max }$.

The pressure and density profiles show only slight differences at the von Neumann state $\left(\mathrm{vN}-x / l_{\text {ind }}=0\right)$ with simplified kinetics overpredicting it. The CJ $\left(x / l_{\text {ind }} \sim 7\right)$ is well captured by all models. The temperature profiles show the opposite behavior as $T$ is higher for detailed chemistry at $\mathrm{CJ}$ and essentially the same at the vN state for all models. The higher value of $T$ at $\mathrm{CJ}$ for detailed chemistry is simply a consequence of accounting for changes in $W$. This can be shown using the ideal gas law: $T_{\mathrm{CJ}}=\left(p / \rho R_{u}\right)_{\mathrm{CJ}} W_{\text {Detailed }}$, since the value of the first term on the right-hand side of the expression is approximately the same for all chemical models. Another evident difference in all the profiles given by simplified kinetics 

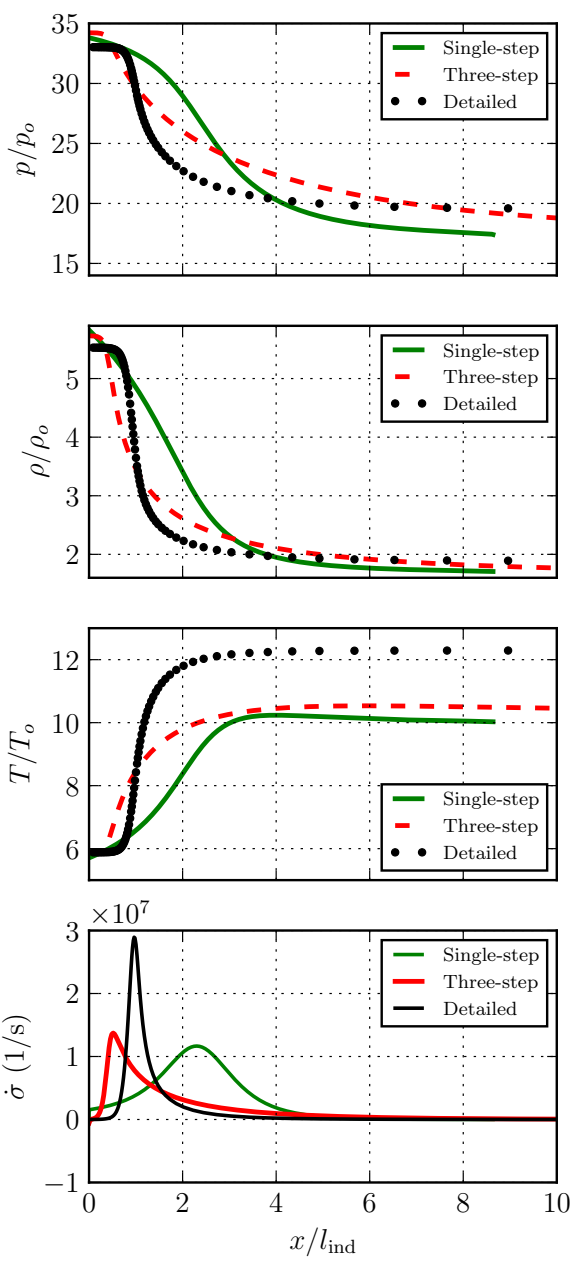

Figure 14: ZND structure obtained using single-step, three-step chain-branching and detailed chemistry. Pressure, density and temperature are normalized by their values in fresh mixture; distance by $l_{\text {ind, Detailed }}=35 \mu \mathrm{m}$. Thermicity, $\dot{\sigma}$, is kept in its dimensional form.

are the trajectories taken to expand the mixture from $\mathrm{vN}$ to $\mathrm{CJ}$.

The thermicity profiles, $\dot{\sigma}$, shed some light as to why this is the case and exhibit the most interesting behavior. The rates at which heat is released, their maxima, as well as the lengths associated to the induction phase and heat release 
differ significantly for simplified kinetics as compared to detailed chemistry. This suggests that the stability of the mixture characterized by the parameter $\chi[46,47]$ is different even though the simplified models are fitted to supposedly mimic the same reactive gas (i.e. stoichiometric $\mathrm{H}_{2}-\mathrm{O}_{2}$ at atmospheric conditions).

The $\chi$ stability parameter $[46,47]$ is defined as $\chi=T_{\mathrm{vN}} / \tau_{\text {ind }} \cdot\left(\partial \tau_{\text {ind }} / \partial T\right)_{\mathrm{vN}} \cdot l_{\text {ind }} / l_{\text {reac }}$ where $l_{\text {reac }}=u_{\mathrm{CJ}} / \dot{\sigma}_{\max }$ is the reaction length; $T_{\mathrm{vN}}$ and $u_{\mathrm{CJ}}$ are the temperature and flow speed at the von Neumann and Chapman-Jouguet state, respectively. To compare the values of $\chi$ among the chemical models, we can write a generic ratio as follows:

$$
\frac{\chi_{a}}{\chi_{b}}=\left(\frac{T_{\mathrm{vN}, a}}{T_{\mathrm{vN}, b}}\right)\left(\frac{\tau_{\text {ind }, b}}{\tau_{\text {ind }, a}}\right)\left(\frac{\partial \tau_{\text {ind }, a}}{\partial \tau_{\text {ind }, b}}\right)\left(\frac{l_{\text {ind }, a}}{l_{\text {ind }, b}}\right)\left(\frac{u_{\mathrm{CJ}, b}}{u_{\mathrm{CJ}, a}}\right)\left(\frac{\dot{\sigma}_{\text {max }, a}}{\dot{\sigma}_{\max , b}}\right)
$$

where $a$ and $b$ are subscripts corresponding to the models being compared. Since the von Neumann temperature and associated delay times, as well as their slopes in the high temperature range are approximately the same for all mechanisms, the first three ratios in Eq. 10 are unity. This simplifies the expression to:

$$
\frac{\chi_{a}}{\chi_{b}} \sim\left(\frac{l_{\text {ind }, a}}{l_{\text {ind }, b}}\right)\left(\frac{u_{\mathrm{CJ}, b}}{u_{\mathrm{CJ}, a}}\right)\left(\frac{\dot{\sigma}_{\max , a}}{\dot{\sigma}_{\max , b}}\right)
$$

The values of the remaining ratios can then be computed using Fig. 14.

$$
\begin{aligned}
& \frac{\chi_{1 \text {-Step }}}{\chi_{\text {3-Step }}} \sim(4.12) \cdot(0.996) \cdot(0.893) \sim 3.664 \\
& \frac{\chi_{\text {Detailed }}}{\chi_{3 \text {-Step }}} \sim(2.06) \cdot(1.115) \cdot(2.176) \sim 4.998 \\
& \frac{\chi_{\text {Detailed }}}{\chi_{1 \text {-Step }}} \sim \frac{\chi_{\text {Detailed }}}{\chi_{3 \text {-Step }}} \cdot \frac{\chi_{3 \text {-Step }}}{\chi_{1 \text {-Step }}} \sim 1.364
\end{aligned}
$$

The results obtained show that while the $\chi$ values for single-step and detailed chemistry are rather close, the differences are large when compared to three-step 
chain-branching chemistry. The discrepancy seems to arise from the differences in $l_{\text {ind }}$ and $\dot{\sigma}_{\max }$ among the mechanisms.

\section{Discussion and conclusion}

Two-dimensional simulations were conducted to assess the influence of the chemical modeling on the detonation structure and its quenching limits. Three different kinetic schemes were used to model a stochiometric $\mathrm{H}_{2}-\mathrm{O}_{2}$ mixture at ambient conditions. Additionally, two configurations were investigated: ideal propagation in a channel and non-ideal semi-confined propagation with eventual quenching.

Three-step chain-branching chemistry reproduces accurately the spectrum of induction times as compared to detailed chemistry, in the high and low temperature regions. Single-step chemistry only captures the high temperature range.

In the ideal case, the range of numerically predicted cell sizes was in agreement with experimental observations, which means that this characteristic length is most probably determined by the high temperature kinetics, as suggested by the phenomenological relations between the cell size and the induction length, computed at the vN state.

The dynamics of the leading shock for both simplified kinetics models and detailed chemistry were similar, as shown by the pdf of the normalized detonation velocity, $D / D_{\mathrm{CJ}}$. Detailed chemistry also shows a flat region around the $\mathrm{CJ}$ value, up to values of $D / D_{\mathrm{CJ}}$ corresponding to the crossover temperature. Below this temperature, the ignition times are no longer commensurate with those associated with adiabatic shock-induced ignition behind detonation fronts. This may explain the presence of bulbs and cusps in the front for three-step chain-branching chem- 
istry. However, this increased curvature is not present in detailed chemistry, which also exhibits reactant consumption downstream of the transverse waves along the front. These are the most remarkable features that could explain the resilience of $\mathrm{H}_{2}$ mixtures to failure.

The critical heights obtained increase as $h_{\text {crit, Detailed }} \ll h_{\text {crit, 3-Step }}<h_{\text {crit, 1-Step. }}$. $h_{\text {crit, Detailed }}$ was by far the lowest among the three chemical models. Although the simplified kinetics schemes and detailed chemistry share the same ignition delays and shock dynamics in the high temperature range, their predicted critical heights differ significantly $\left(h_{\text {crit, 3-Step }} / h_{\text {crit, Detailed }} \sim 3.33\right.$ ). The critical height for single-

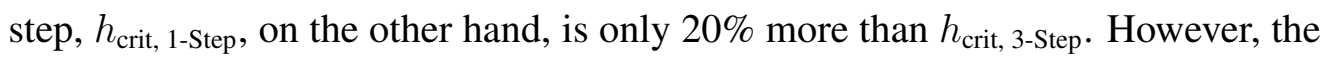
excursion of the detonation wave to low velocities and corresponding post-shock temperatures below the crossover temperature should favor quenching to occur at a height lower than that of the single-step model whose induction time is shorter. Another differentiating feature between the simplified models in this temperature range is that for three-step chain-branching chemistry the rate of production of radicals decreases significantly and heat release to the mixture stops abruptly. This is in contrast with single-step chemistry where energy deposition continues over a longer spatial scale as evidenced from the thermicity profiles. In real $\mathrm{H}_{2}$ systems however, there is a change in chemical pathways for $T<T_{B}$ where linear chains take over, partially converting the $\mathrm{H}_{2}$ to $\mathrm{HO}_{2}$ that in the presence of enough $\mathrm{H}$ atoms can replenish the radical pool through $\mathrm{H}+\mathrm{HO}_{2} \rightarrow \mathrm{OH}+\mathrm{OH}$, ultimately resulting in ignition of the mixture and associated heat release.

Analysis of the induction times of different samples within an unburnt pocket indicates that the presence of active radicals cannot solely explain the observed resistance to losses. As hydrogen is consumed, the molecular weight increases 
resulting in temperature rising at a higher rate within the reaction zone than in the simplified kinetics models. This results in enhanced reactant consumption. Nevertheless, the effective activation energy of the mixture is reduced whose regularizing effect may not completely explain the emergence of reactive transverse waves.

In terms of $\chi$ the mixture stability increases (values of $\chi$ decrease) as $\chi_{\text {Detailed }}>$ $\chi_{1 \text {-Step }} \gg \chi_{3 \text {-Step }}$. The increase of $\chi$ (or equivalently of the reduced activation energy in the case of single-step models) and associated instabilities are not sufficient to explain the higher critical height. However, the $h_{\text {crit }}$ results seem to correlate well with the values of $\dot{\sigma}_{\max }$ obtained, i.e. $\dot{\sigma}_{\max } / \dot{\sigma}_{\max , \text { Detailed }} \propto h_{\text {crit }} / h_{\text {crit, Detailed. }}$. This suggests that improved simplified models could be developed by using the thermicity profiles together with ignition delay times as fitting targets.

Furthermore, due to the hydrogen depletion, the ratio of specific heats $\gamma$ approaches one, crossing over the critical value [48] for which inert and reactive shock bifurcations give rise to the spontaneous appearance of triple points [49, 50]. These gasdynamics features together with the transverse reactive waves observed in the detailed chemistry results may provide the necessary feedback to promote fuel consumption within unburnt pockets, and ultimately account for its observed resistance to losses.

The critical height predicted with detailed chemistry $\left(h_{\text {crit }}=6 \mathrm{~mm}\right)$ is in good agreement with experimental data $\left(h_{\text {crit }} \simeq 4.6 \mathrm{~mm}\right)$ [18], despite the fact that we neglect three-dimensional effects. This advocates for the predictive capabilities of detailed chemistry as compared to simplified kinetics. Nonetheless, we acknowledge that this outcome is specific to $\mathrm{H}_{2}$ mixtures and direct extrapolation of our results to hydrocarbons may not be applicable as their cellular structure is very ir- 
regular. For the latter it may be necessary to assess whether diffusive consumption of unburnt pockets plays a role.

A natural extension of our work would be to allow the three-step chain-branching model to include molecular weight and ratio of specific heats variations; also, extension of the simplified kinetics to include competition for radicals as discussed in [51] may provide the missing physics to improve the predictive capabilities of simplified models.

\section{Acknowledgements}

The computations were performed using HPC resources from the cluster of Pprime, the Mésocentre de Calcul Poitevin and from GENCI-CINES (Grant 2018A0052B07735). This work was supported by the CPER FEDER Project of Région Nouvelle Aquitaine.

\section{References}

[1] Y. B. Zel'dovich, On the theory of detonation propagation in gaseous systems, Zh. Eksp. Teor. Fiz 10 (1940) 542-568.

[2] J. H. S. Lee, M. I. Radulescu, On the hydrodynamic thickness of cellular detonations, Combustion, Explosion and Shock Waves 41 (2005) 745-765.

[3] M. I. Radulescu, G. J. Sharpe, C. K. Law, J. H. Lee, The hydrodynamic structure of unstable cellular detonations, Journal of Fluid Mechanics 580 (2007) 31-81.

[4] R. A. Strehlow, Nature of transverse waves in detonations, Astronautica Acta 14 (1969) 539. 
[5] C. Brochet, Contribution à l'étude des détonations instables dans les mélanges gazeux, Ph.D. thesis, Poitiers University, 1966.

[6] M. I. Radulescu, B. Borzou, Dynamics of detonations with a constant mean flow divergence, Journal of Fluid Mechanics 845 (2018) 346-377.

[7] W. P. Sommers, R. B. Morrison, Simulation of condensed explosive detonation phenomena with gases, Physics of Fluids 5 (1962) 241-248.

[8] A. Teodorczyk, J. H. S. Lee, Detonation attenuation by foams and wire meshes lining the walls, Shock Waves 4 (1995) 225-236.

[9] M. I. Radulescu, J. H. S. Lee, The failure mechanism of gaseous detonations: experiments in porous wall tubes, Combustion and Flame 131 (2002) 29 46.

[10] M. Reynaud, F. Virot, A. Chinnayya, Interactions of a detonation wave confined by a high-temperature compressible layer, in: 26th ICDERS meeting, Boston, USA., pp. 1-6.

[11] M. Reynaud, Numerical Study of Detonation Confined by an Inert Gas, Ph.D. thesis, ISAE-ENSMA Ecole Nationale Supérieure de Mécanique et d'Aérotechique - Poitiers, France, 2017.

[12] R. W. Houim, R. T. Fievisohn, The influence of acoustic impedance on gaseous layered detonations bounded by an inert gas, Combustion and Flame 179 (2017) 185-198.

[13] X. Mi, A. Higgins, C. Kiyanda, H. Ng, N. Nikiforakis, Effect of spatial in- 
homogeneities on detonation propagation with yielding confinement, Shock Waves 28 (2018) 993-1009.

[14] C. Chiquete, M. Short, J. J. Quirk, The effect of curvature and confinement on gas-phase detonation cellular stability, Proceedings of the Combustion Institute 37 (2019) 3565-3573.

[15] W. Rudy, M. Kuznetsov, R. Porowski, A. Teodorczyk, J. Grune, K. Sempert, Critical conditions of hydrogen-air detonation in partially confined geometry, Proceedings of the Combustion Institute 34 (2013) 1965-1972.

[16] W. P. Sommers, The interaction of a detonation wave with an inert boundary, Ph.D. thesis, University of Michigan, Ann Arbor, 1961.

[17] E. K. Dabora, The influence of a compressible boundary on the propagation of gaseous detonations, Technical Report 3559-E, NASA, 1963.

[18] E. K. Dabora, J. A. Nicholls, R. B. Morrison, The influence of a compressible boundary on the propagation of gaseous detonations, $10^{\text {th }}$ Symposium (International) on Combustion 10 (1965) 817 - 830.

[19] S. B. Murray, J. Lee, The influence of yielding confinement on large-scale ethylene-air detonations, Prog. Astronaut. Aeronaut 94 (1984) 80-103.

[20] W. Rudy, M. Zbikowski, A. Teodorczyk, Detonations in hydrogen-methaneair mixtures in semi confined flat channels, Energy 116 (2016) 1479-1483.

[21] W. Rudy, K. Dziubanii, M. Zbikowski, A. Teodorczyk, Experimental determination of critical conditions for hydrogen-air detonation propagation in 
partially confined geometry, International Journal of Hydrogen Energy 42 (2017) 7366-7373.

[22] J. Grune, K. Sempert, A. Friedrich, M. Kuznetsov, T. Jordan, Detonation wave propagation in semi-confined layers of hydrogen-air and hydrogenoxygen mixtures, International Journal of Hydrogen Energy 42 (2017) 7589-7599.

[23] M. I. Radulescu, A detonation paradox: Why inviscid detonation simulations predict the incorrect trend for the role of instability in gaseous cellular detonations?, Combustion and Flame (2018).

[24] M. I. Radulescu, G. J. Sharpe, J. H. S. Lee, C. B. Kiyanda, A. J. Higgins, R. K. Hanson, The ignition mechanism in irregular structure gaseous detonations, Proceedings of the Combustion Institute 30 (2005) 1859-1867.

[25] M. A. Birkan, D. R. Kassoy, The unified theory for chain branching thermal explosions with dissociation-recombination and confinement effects, Combustion Science and Technology 44 (1986) 223-256.

[26] M. Short, J. J. Quirk, On the nonlinear stability and detonability limit of a detonation wave for a model three-step chain-branching reaction, Journal of Fluid Mechanics 339 (1997) 89-119.

[27] B. Varatharajan, F. Williams, Chemical-kinetic descriptions of hightemperature ignition and detonation of acetylene-oxygen-diluent systems, Combustion and Flame 124 (2001) 624-645.

[28] G. J. Sharpe, N. Maflahi, Homogeneous explosion and shock initiation for a 
three-step chain-branching reaction model, Journal of Fluid Mechanics 566 (2006) 163-194.

[29] J. Melguizo-Gavilanes, L. Bauwens, A comparison between constant volume induction times and results from spatially resolved simulation of ignition behind reflected shocks: implications for shock tube experiments, Shock Waves 23 (2013) 221-231.

[30] R. Mével, J. Sabard, J. Lei, N. Chaumeix, Fundamental combustion properties of oxygen enriched hydrogen/air mixtures relevant to safety analysis: Experimental and simulation study, international journal of hydrogen energy 41 (2016) 6905-6916.

[31] S. Gallier, F. Le Palud, F. Pintgen, R. Mével, J. Shepherd, Detonation wave diffraction in $\mathrm{H} 2-\mathrm{O} 2-\mathrm{Ar}$ mixtures, Proceedings of the Combustion Institute 36 (2017) 2781-2789.

[32] A. Suresh, H. Huynh, Accurate monotonicity-preserving schemes with Runge-Kutta time stepping, Journal of Computational Physics 136 (1997) 83-99.

[33] S. Xu, T. Aslam, D. S. Stewart, High resolution numerical simulation of ideal and non-ideal compressible reacting flows with embedded internal boundaries, Combustion Theory and Modelling (1997).

[34] A. Mignone, P. Tzeferacos, G. Bodo, High-order conservative finite difference GLM-MHD schemes for cell-centered MHD, Journal of Computational Physics 229 (2010) 5896-5920. 
[35] E. F. Toro, M. Spruce, W. Speares, Restoration of the contact surface in the HLL-riemann solver, Shock waves 4 (1994) 25-34.

[36] Z. Shen, W. Yan, G. Yuan, A robust HLLC-type riemann solver for strong shock, Journal of Computational Physics 309 (2016) 185-206.

[37] A. Sow, A. Chinnayya, A. Hadjadj, On the viscous boundary layer of weakly unstable detonations in narrow channels, Computers \& Fluids 179 (2019) 449-458.

[38] M. Reynaud, F. Virot, A. Chinnayya, A computational study of the interaction of gaseous detonations with a compressible layer, Physics of Fluids 29 (2017) 056101.

[39] B. Savard, Y. Xuan, B. Bobbitt, G. Blanquart, A computationally-efficient, semi-implicit, iterative method for the time-integration of reacting flows with stiff chemistry, Journal of Computational Physics 295 (2015) 740-769.

[40] C. Kiyanda, A. Higgins, Photographic investigation into the mechanism of combustion in irregular detonation waves, Shock Waves 23 (2013) 115-130.

[41] B. M. Maxwell, R. R. Bhattacharjee, S. S. Lau-Chapdelaine, S. A. Falle, G. J. Sharpe, M. I. Radulescu, Influence of turbulent fluctuations on detonation propagation, Journal of Fluid Mechanics 818 (2017) 646-696.

[42] B. Taylor, D. Kessler, V. Gamezo, E. Oran, The influence of chemical kinetics on the structure of hydrogen-air detonations, in: 50th AIAA Aerospace Sciences Meeting including the New Horizons Forum and Aerospace Exposition, p. 979. 
[43] M. Kaneshige, J. E. Shepherd, Detonation database: GALCIT Report FM978, California Institute of Technology (1997).

[44] L. Shi, H. Shen, P. Zhang, D. Zhang, C. Wen, Assessment of vibrational non-equilibrium effect on detonation cell size, Combustion Science and Technology 189 (2017) 841-853.

[45] R. Mével, S. Gallier, Structure of detonation propagating in lean and rich dimethyl ether-oxygen mixtures, Shock Waves 28 (2018) 955-966.

[46] H. D. Ng, Y. Ju, J. H. Lee, Assessment of detonation hazards in highpressure hydrogen storage from chemical sensitivity analysis, International Journal of Hydrogen Energy 32 (2007) 93-99.

[47] M. I. Radulescu, The propagation and failure mechanism of gaseous detonations: experiments in porous-walled tubes, Ph.D. thesis, McGill University Libraries, 2003.

[48] P. Mach, M. Radulescu, Mach reflection bifurcations as a mechanism of cell multiplication in gaseous detonations, Proceedings of the Combustion Institute 33 (2011) 2279-2285.

[49] S.-M. Lau-Chapdelaine, M. I. Radulescu, Viscous solution of the tripleshock reflection problem, Shock Waves 26 (2016) 551-560.

[50] S. S.-M. Lau-Chapdelaine, Viscous triple shock reactions relevant to detonation waves, and Detonation dynamics predicted by the Fickett model, Ph.D. thesis, University of Ottawa, Canada, 2019. 
[51] Z. Liang, S. Browne, R. Deiterding, J. Shepherd, Detonation front structure and the competition for radicals, Proceedings of the Combustion Institute 31 (2007) 2445-2453.

[52] V. N. Gamezo, D. Desbordes, E. S. Oran, Formation and evolution of twodimensional cellular detonations, Combustion and Flame 116 (1999) 154165.

[53] V. Gamezo, D. Desbordes, E. Oran, Two-dimensional reactive flow dynamics in cellular detonation waves, Shock Waves 9 (1999) 11-17.

[54] J. Lipkowicz, I. Wlokas, A. Kempf, Analysis of mild ignition in a shock tube using a highly resolved 3D-LES and high-order shock-capturing schemes, Shock Waves 29 (2019) 511-521. 


\section{Appendix A. Numerical convergence}

A convergence study was performed to determine an adequate spatial resolution to run our simulations. Here, we only show results for three-step chainbranching and detailed chemistry because the numerical convergence for singlestep has been thoroughly presented in previous work by our group using the same in-house code [38]. The computational domain used is a channel of $L_{x}=100$ $\mathrm{mm}$ by $L_{y}=2 \mathrm{~mm}$ corresponding to $\sim 120 l_{\text {ind }}$ and $\sim 60 l_{\text {ind }}$ along the height of the channel for three-step chain-branching and detailed chemistry, respectively. Four resolutions were tested: $2,5,10$ and 20 points per induction length $l_{\text {ind }}$. The detonations were initiated by placing a circular region at high pressure and temperature $\left(p_{\mathrm{ign}} / p_{o} \sim 34 ; \rho_{\mathrm{ign}} / \rho_{o} \sim 6\right)$. A blast wave forms at early stages that reflects from the top and bottom wall generating a shock-shock interaction near the center of the channel that transitions into self-sustained detonations. See the numerical soot foils presented in Figs. A.1 and A.2.

Results are shown for the first $60 \mathrm{~mm}$ of the channel. Detailed chemistry (Fig. A.1) displays clearly the cell size evolution as the resolution is increased, starting from a very regular structure at $l_{\text {ind }} / 2$, gradually admitting additional length scales as more points are included in $l_{\text {ind }}$. For all resolutions considered the detonations undergo two phases: (i) a first transient where the cells are smaller and regular; a typical feature of overdriven detonations. For higher resolutions this zone becomes progressively smaller going from $40 \mathrm{~mm}$ at $l_{\text {ind }} / 5$ to $15 \mathrm{~mm}$ at $l_{\text {ind }} / 10$. This evolution is in line with the discussion in Gamezo et al. [52, 53]. Results for $l_{\text {ind }} / 10$ and $l_{\text {ind }} / 20$ yield approximately the same length for the initial phase. (ii) a second transient where the detonations relax to a steady propagation velocity and approximately the same level of irregularities in their cellular struc- 
tures. To asses the effect of the mode of initiation, the center of the high pressure and temperature region was offset. The simulations for $l_{\text {ind }} / 2$ and $l_{\text {ind }} / 5$ were repeated with this configuration; no appreciable changes were obtained providing evidence of initiation mode independence.

For three-step chain-branching chemistry (Fig. A.2), in contrast with detailed chemistry, the length of the initial transient is approximately the same for all resolutions; the cellular structure expectedly shows additional irregularities as the resolution is increased. The detonation dynamics, cell regularity and sizes remain essentially unchanged when the number of points in $l_{\text {ind }}$ is doubled from 10 to 20. Previous studies using high-order difference schemes for the study of detonations [38, 13] and mild ignition [54] have reported similar resolution requirements. 


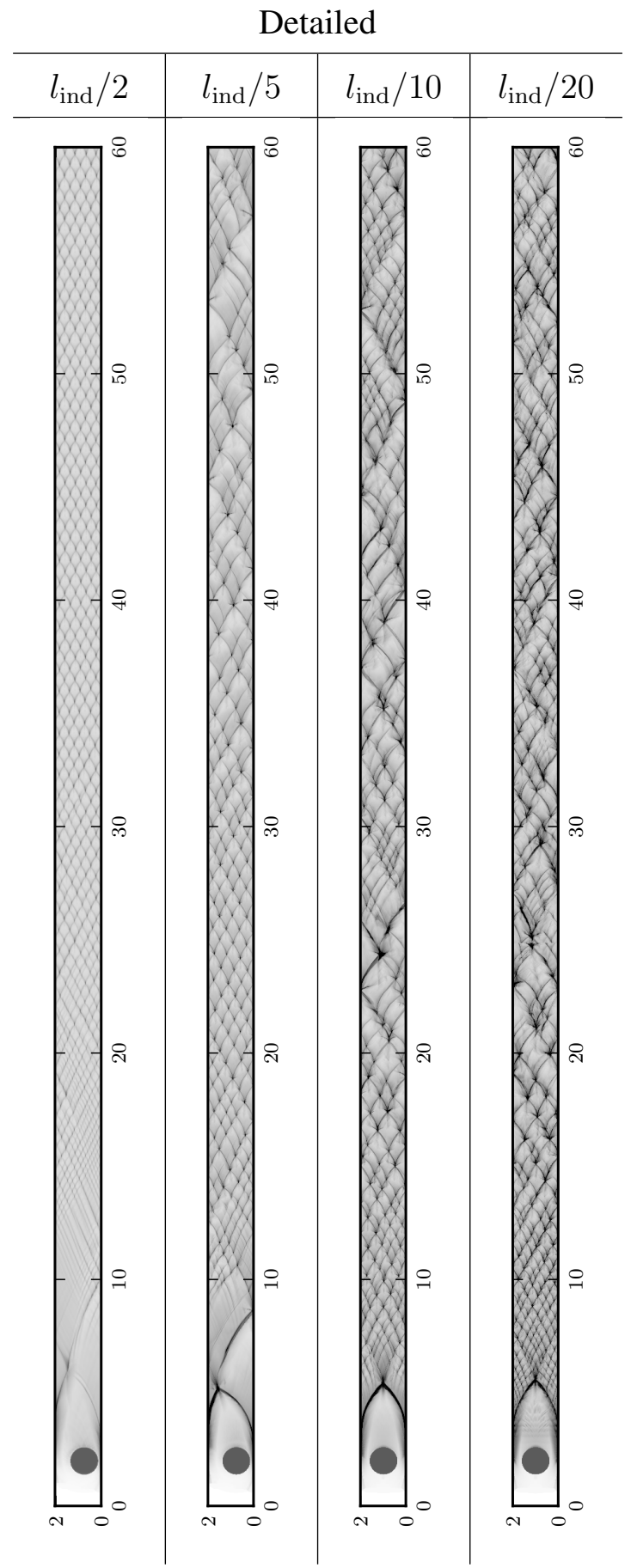

Figure A.1: Numerical soot foils for detailed chemistry as a function of increasing resolution $\left(\Delta x=l_{\text {ind }} / k, k=2,5,10\right.$ and 20$)$. Distances are in $\mathrm{mm}$. 


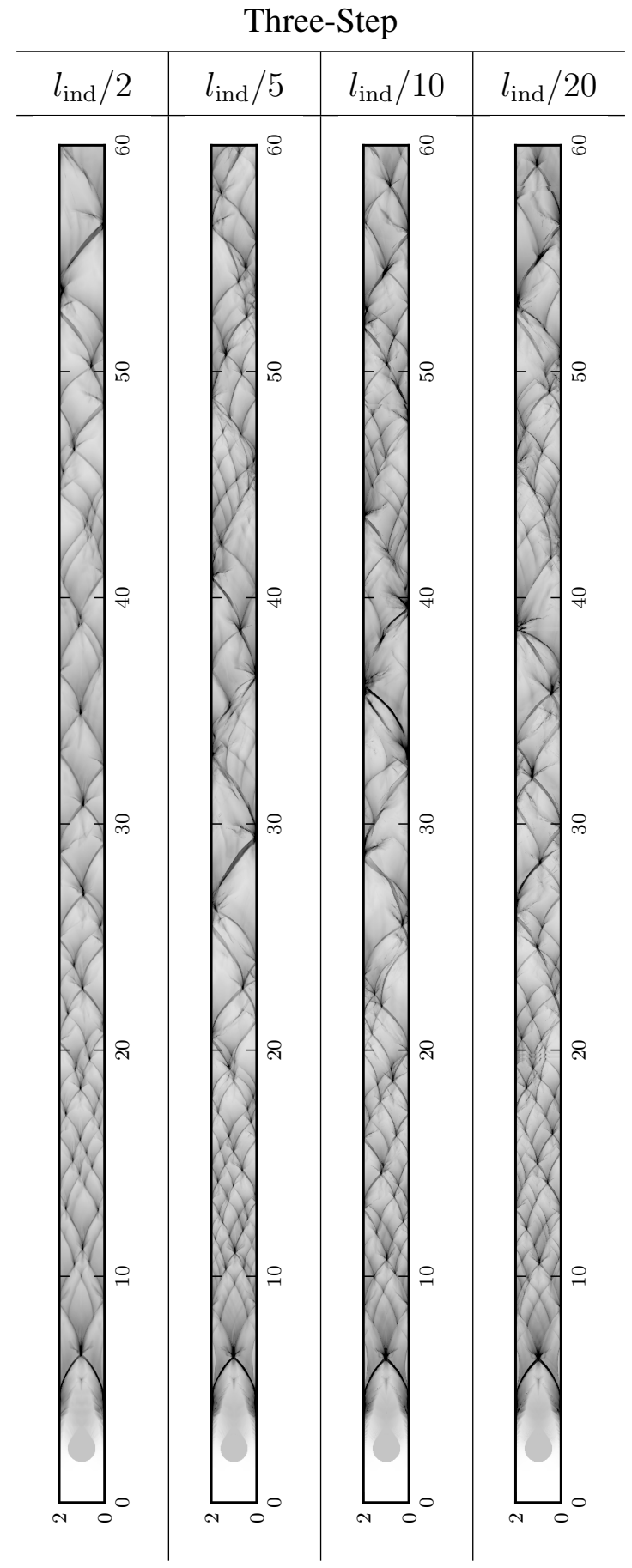

Figure A.2: Numerical soot foils for three-step chain-branching chemistry as a function of increasing resolution ( $\Delta x=l_{\text {ind }} / k, k=2,5,10$ and 20 ). Distances are in $\mathrm{mm}$. 